\title{
Percutaneous radiofrequency ablation for clinical stage I non-small cell lung cancer: Results in 20 nonsurgical candidates
}

Takao Hiraki, MD, ${ }^{\text {a }}$ Hideo Gobara, MD, ${ }^{a}$ Tatsuhiko lishi, MD, ${ }^{a}$ Yoshifumi Sano, MD, ${ }^{\mathrm{b}}$ Toshihiro Iguchi, MD, ${ }^{a}$ Hiroyasu Fujiwara, MD, ${ }^{a}$ Nobuhisa Tajiri, MD, ${ }^{a}$ Jun Sakurai, MD, ${ }^{a}$ Hiroshi Date, MD, ${ }^{b}$ Hidefumi Mimura, MD, ${ }^{a}$ and Susumu Kanazawa, $\mathrm{MD}^{\mathrm{a}}$

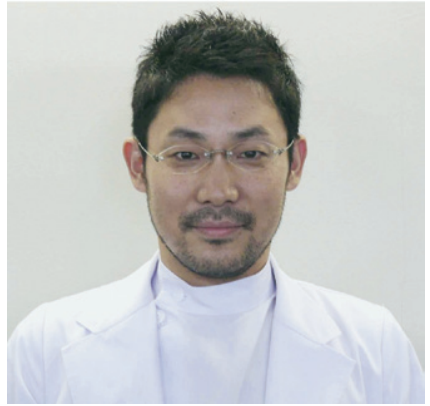

Dr Hiraki
From the Departments of Radiology a and Cancer and Thoracic Surgery, ${ }^{\mathrm{b}}$ Okayama University Medical School, Okayama, Japan.

Received for publication March 14, 2007; revisions received July 4, 2007; accepted for publication July 10, 2007.

Address for reprints: Takao Hiraki, MD, 2-5-1 Shikatacho, Okayama 700-8558, Japan (E-mail: takaoh@tc4.so-net.ne.jp).

J Thorac Cardiovasc Surg 2007;134:1306-12 $0022-5223 / \$ 32.00$

Copyright $\odot 2007$ by The American Association for Thoracic Surgery

doi:10.1016/j.jtcvs.2007.07.013
Objective: Our objective was to evaluate the outcomes of radiofrequency ablation for nonsurgical candidates with clinical stage I non-small cell lung cancer.

Methods: This study was carried out on 20 nonsurgical candidates (11 men and 9 women; mean age, 75.6 years) with clinical stage I (IA, $\mathrm{n}=14 ; \mathrm{IB}, \mathrm{n}=6$ ) non-small cell lung cancer. All patients were deemed nonsurgical candidates because of poor pulmonary function $(n=7)$, poor cardiac function $(n=3)$, poor performance status $(n=4)$, or refusal to undergo surgery $(n=6)$. The mean long-axis tumor diameter was $2.4 \mathrm{~cm}$. Twenty patients underwent 23 percutaneous ablation sessions, including 3 repeat sessions for the treatment of local progression. The outcomes of radiofrequency ablation were retrospectively evaluated.

Results: Procedural complications included pneumothorax $(\mathrm{n}=13$ [57\%]) and pleural effusion $(\mathrm{n}=4[17 \%])$. One case of pneumothorax required chest tube placement. The median follow-up period was 21.8 months. Local progression was observed in 7 (35\%) patients a median of 9.0 months after the first session. The local control rates were $72 \%$ at 1 year, $63 \%$ at 2 years, and $63 \%$ at 3 years. The mean survival time was 42 months. The overall survivals and cancer-specific survivals were $90 \%$ and $100 \%$ at 1 year, $84 \%$ and $93 \%$ at 2 years, and $74 \%$ and $83 \%$ at 3 years, respectively.

Conclusions: Treating clinical stage I non-small cell lung cancer with one or more radiofrequency ablation sessions provided promising outcomes in terms of survival, although the rate of local progression was relatively high. A prospective study with a larger population and longer follow-up is warranted.

$\mathrm{P}$ rimary lung cancer is the most common malignant disease and the leading cause of death from cancer worldwide. Surgical resection is the first-line treatment and the best option for treating early-stage non-small cell lung cancer (NSCLC). Although some surgeons believe that limited resection is effective in treating localized cancer, lobectomy is considered to be the gold standard of surgery because a large randomized controlled trial demonstrated the superiority of lobectomy as compared with limited resection in terms of both patient survival and locoregional recurrence in T1 N0 NSCLC. ${ }^{1}$ Unfortunately, certain patients are considered medically inoperable. Conventional external beam radiation therapy (XRT) has been traditionally administered to such patients; the outcomes, however, are suboptimal. Thus, various alternative modalities, including radiofrequency ablation (RFA), have been the focus of many studies.

RFA is a thermal therapy that results in coagulation necrosis. The procedure involves placement of an electrode into the target followed by application of radiofrequency energy. Initially, RFA was mainly used for treating hepatocellular 


$$
\begin{aligned}
& \text { Abbreviations and Acronyms } \\
& \begin{aligned}
\text { CT } & =\text { computed tomography } \\
\text { NSCLC } & =\text { non-small cell lung cancer } \\
\text { PET } & =\text { positron emission tomography } \\
\text { RFA } & =\text { radiofrequency ablation } \\
\text { XRT } & =\text { external beam radiation therapy }
\end{aligned}
\end{aligned}
$$

carcinoma. The favorable outcomes in the treatment of hepatocellular carcinoma have encouraged the application of this technique to neoplasms in other organs, including the lungs. Preliminary studies of RFA in treating lung tumors have shown promising results for initial local control. ${ }^{2,3}$ The purpose of this study was to retrospectively evaluate the outcomes of RFA when used to treat nonsurgical candidates with clinical stage I NSCLC.

\section{Patients and Methods \\ Study Population}

Approval from the institutional review board and informed consents from the patients were obtained to perform RFA of lung tumors. From June 2001 to February 2006, we treated 209 patients having primary lung cancer $(n=40)$ or metastatic lung neoplasms from various primary sites $(n=169)$ with 329 percutaneous RFA sessions at our institution. Of the 40 patients with primary lung cancer, 20 were excluded from the study: 18 had clinical stage II cancer or higher and were treated with a combination of RFA and systemic chemotherapy, and 2 had clinical stage I cancer but were treated by a combination of RFA and XRT owing to a large tumor size. Clinical stage I NSCLC was diagnosed in the remaining 20 patients, and they were treated by RFA alone; these patients formed the basis of our study. For clinical staging, chest and abdominal computed tomographic (CT) scans were performed in all patients. Further, brain magnetic resonance imaging and bone scintiscan were performed in 6 patients and in 2 patients, respectively. Because our institution was not equipped with positron emission tomography (PET) facilitates, none of the patients underwent PET scanning except for 3 patients who underwent it at another institution. None of the patients underwent mediastinoscopy, endoscopic ultrasound-guided fine needle aspiration, or endoscopic bronchial ultrasonography for the examination of lymph node involvement. Thus, lymph node involvement was evaluated on CT images, and lymph node metastasis was considered absent because none of the lymph nodes was larger than $1.0 \mathrm{~cm}$ in short-axis diameter.

The characteristics of the 20 patients and the tumors are summarized in Table 1. In brief, there were 11 men and 9 women (mean age, 75.6 years; range, $64-88$ years). Of the 20 patients, 13 had adenocarcinoma including 3 patients with lesions showing pure ground-glass opacity and 7 had squamous cell carcinoma. Clinical stage IA and IB cancers were diagnosed in 14 and 6 patients, respectively. The mean long-axis tumor diameter was 2.4 $\mathrm{cm}$ (median, $2.0 \mathrm{~cm}$; range, $1.3-6.0 \mathrm{~cm}$ ). The patients were first

\begin{tabular}{|c|c|c|c|c|c|c|c|c|c|}
\hline $\begin{array}{l}\text { Patient } \\
\text { no. }\end{array}$ & $\begin{array}{l}\text { Age } \\
\text { (yr) }\end{array}$ & Sex & Pathology & $\begin{array}{l}\text { Size } \\
\text { (cm) }\end{array}$ & Stage & $\begin{array}{c}\text { Follow-up } \\
\text { (mo) }\end{array}$ & $\begin{array}{l}\text { Patient's } \\
\text { outcome }\end{array}$ & Cause of death & $\begin{array}{l}\text { Recurrence at latest or } \\
\text { last follow-up }\end{array}$ \\
\hline 1 & 79 & Male & Adeno & 1.5 & $\mathrm{IA}$ & 52 & Alive & & Local \\
\hline 2 & 81 & Male & Squamous & 2 & IA & 2 & Death & Coronary disease & None \\
\hline 3 & 88 & Female & Squamous & 2.8 & IB & 30.8 & Alive & & None \\
\hline 4 & 79 & Male & Squamous & 3.7 & IB & 35.7 & Alive & & None \\
\hline 5 & 81 & Male & Squamous & 6 & IB & 13.4 & Death & Cancer progression & Local \\
\hline 6 & 80 & Female & Adeno & 1.6 & $\mathrm{IA}$ & 38.3 & Alive & & Local \\
\hline 7 & 65 & Female & Squamous & 2 & IA & 24.5 & Death & Cancer progression & Local, intrapulmonary \\
\hline 8 & 77 & Male & Squamous & 1.6 & IA & 30.2 & Alive & & None \\
\hline 9 & 67 & Male & Squamous & 2.1 & IA & 26.2 & Alive & & $\begin{array}{l}\text { Local, lymph node, } \\
\text { intrapulmonary }\end{array}$ \\
\hline 10 & 79 & Male & Adeno & 3.2 & IB & 2.8 & Death & Respiratory failure & None \\
\hline 11 & 78 & Female & Adeno & 2.5 & $\mathrm{IA}$ & 29.7 & Alive & & None \\
\hline 12 & 77 & Female & Adeno & 1.3 & $\mathrm{IA}$ & 30.1 & Alive & & None \\
\hline 13 & 67 & Male & Adeno & 3 & IB & 17.8 & Alive & & None \\
\hline 14 & 77 & Female & Adeno & 3.4 & IB & 24.1 & Alive & & None \\
\hline 15 & 65 & Female & Adeno & 1.5 & $\mathrm{IA}$ & 19.5 & Alive & & None \\
\hline 16 & 73 & Male & Adeno & 1.6 & IA & 17.9 & Alive & & None \\
\hline 17 & 72 & Male & Adeno & 1.7 & IA & 13.2 & Alive & & None \\
\hline 18 & 64 & Male & Adeno & 1.8 & IA & 11.9 & Alive & & None \\
\hline 19 & 78 & Female & Adeno & 1.4 & IA & 12.4 & Alive & & None \\
\hline 20 & 84 & Female & Adeno & 2.3 & $\mathrm{IA}$ & 12.2 & Alive & & Local, intrapulmonary \\
\hline Average & 75.6 & & & 2.4 & & 22.2 & & & \\
\hline
\end{tabular}
referred to the department of thoracic surgery. All patients were determined to be nonsurgical candidates by a surgeon because of poor pulmonary function (predicted forced respiratory volume in 1 second $\leq 1000 \mathrm{~mL}, \mathrm{n}=7$ ), poor cardiac function (New York

TABLE 1. Patient and tumor characteristics and follow-up results 
Heart Association Class $\geq$ III, $\mathrm{n}=3$ ), poor performance status (status $\geq 2, n=4)$, or refusal to undergo surgery $(n=6)$. The mean forced respiratory volume in 1 second in the 7 patients with poor pulmonary function was 1.181 (range, 0.97-1.45 1). All 20 patients underwent 23 RFA sessions, including 3 repeat sessions for the treatment of local progression after a previous session. None of these patients underwent adjuvant systemic chemotherapy.

\section{RFA Techniques}

The electrodes that were used in the 23 sessions included a multitined expandable electrode (LeVeen; Boston Scientific, Natick, Mass) with a 2-cm diameter array $(\mathrm{n}=9)$; a single internally cooled electrode (Cool-tip; Valleylab, Boulder, Colo) with a $1-\mathrm{cm}(\mathrm{n}=4), 2-\mathrm{cm}(\mathrm{n}=8)$, or $3-\mathrm{cm}(\mathrm{n}=1)$ noninsulated tip; or an internally cooled cluster electrode $(n=1)$ (Cool-tip). Until October 2003, only the internally cooled electrode was available at our institution, and therefore this electrode was used in all procedures. Thereafter, the multitined expandable electrodes were made available. The procedure was always performed percutaneously with CT fluoroscopy. Intraprocedural pain was treated by local anesthesia $(n=20)$ or epidural anesthesia $(n=3)$.

The patients were placed in the supine or prone positions depending on the tumor location, and grounding pads were placed on their thighs. After administration of anesthesia, the electrode was introduced into the tumor and was connected to a generator. In the case of the Valleylab device, radiofrequency energy was applied with an impedance control algorithm for 12 minutes during internal cooling of the electrode. Thereafter, the temperature was measured at the electrode tip; if it had not reached $60^{\circ} \mathrm{C}$, another energy application was added at the same site. When the Boston Scientific device was being used, the energy was applied until the impedance showed a rapid increase or an automatic shutoff occurred at 15 minutes; this was repeated once at each site. The ablative margin was obtained by creating multiple overlapping ablation zones when using an electrode with an array diameter or a noninsulated tip length that was equal to or smaller than the tumor diameter.

\section{Follow-up}

The patients were followed up, whenever possible, at 1, 3, 6, 9, and 12 months and thereafter at 6-month intervals. As a result, all 20 patients were followed up at 1 month. A certain number of patients should have undergone subsequent periodic follow-up examinations, that is, 18,17 , and 15 patients at 3 and 6,9 , and 12 months, respectively. Of these, 17,13 , and 13 patients were followed up at 3 and 6, 9, and 12 months, respectively. Further, all of the 11 patients who should have undergone follow-up at 6-month intervals after 12 months were successfully followed up. At every follow-up, chest CT scan was performed to assess the RFA outcomes. The images were obtained before and 30 and 90 seconds after the initiation of intravenous contrast administration at a rate of $3 \mathrm{~mL} / \mathrm{s}$. For assessment of hematogenous metastasis, abdominal CT scanning was generally performed at 6-month intervals. A total of 4 PET examinations were also performed for 3 patients; those were done at 2 months $(n=1), 4$ months $(n=2)$, and 8 months $(\mathrm{n}=1)$. When symptoms suggesting brain or bone metastasis were observed, we performed brain magnetic resonance imaging or bone scintiscan, respectively.

Initial efficacy was assessed on CT images at 1 month; the tumor was considered to be treated if it was entirely covered by the ablation zone. As described in a previous study, in the first 3 months, size of the ablated lesion usually exceeds that of the tumor before ablation even if the tumor is completely ablated; this is because the zone of ablation includes the tumor and the ablated margin of parenchyma to a certain extent. ${ }^{4}$ Hence, during this period, the effectiveness of RFA can be determined not by comparing the tumor size but by assessing the contrast enhancement. The tumor was considered to be completely treated when contrast enhancement was not observed in the entire ablation zone or when the ablation zone exhibited a peripheral rim of contrast enhancement that was concentric, symmetric, and uniform with smooth inner margins. Such an enhancement zone is considered to correspond to reactive hyperemia, inflammation, or granulation at the marginal parenchyma. ${ }^{4}$ After the first 3 months, the ablation zone exhibits gradual involution over time when the tumor is completely ablated. ${ }^{4}$ The appearance of irregular, scattered, nodular, or eccentric foci in the ablation zones was considered to indicate the local progression of the tumor. ${ }^{4}$ The focus generally exhibited contrast enhancement, thereby contrasting against the noncontrastenhanced necrotic tumor tissue. Local progression was also indicated when the ablation zone was circumferentially enlarged; such a zone was generally accompanied by contrast enhancement.

Local control was defined as no evidence suggesting local progression by a first session. The local control rates and overall and cancer-specific survivals were estimated by the Kaplan-Meier analysis. Further, survivals were also estimated according to the cancer stage (IA or IB).

\section{Results}

Among the 23 sessions, procedural complications included pneumothorax $(n=13[57 \%])$ and pleural effusion $(n=4$ [17\%]). One patient with pneumothorax required chest tube placement; all other patients with pneumothoraces and pleural effusion were treated conservatively. No hemorrhagic event requiring treatment was observed, and none of the patients died as a result of this procedure. The median hospital stay was 4 days (range, 2-17 days). The follow-up results are summarized in Table 1 . The median follow-up period was 21.8 months (range, 2-52 months). In all the patients, the tumor was considered to be treated with initial efficacy on CT images at 1 month. Local progression was observed in $7(35 \%)$ patients at 4.4 to 38.3 months (mean, 13.6 months; median, 9.0 months) after the first session. In 2 of these patients, biopsy was performed to confirm the local progression; the remaining patients did not undergo biopsy because local progression was obvious on CT images. Three PET examinations at 2 or 4 months showed positive results, which were in contrast to the results on CT images at the same period; the tumors were then monitored by CT and have not shown local progression. One PET at 8 months showed a negative result, which was in accordance with the result on $\mathrm{CT}$; the tumor was then monitored by CT 


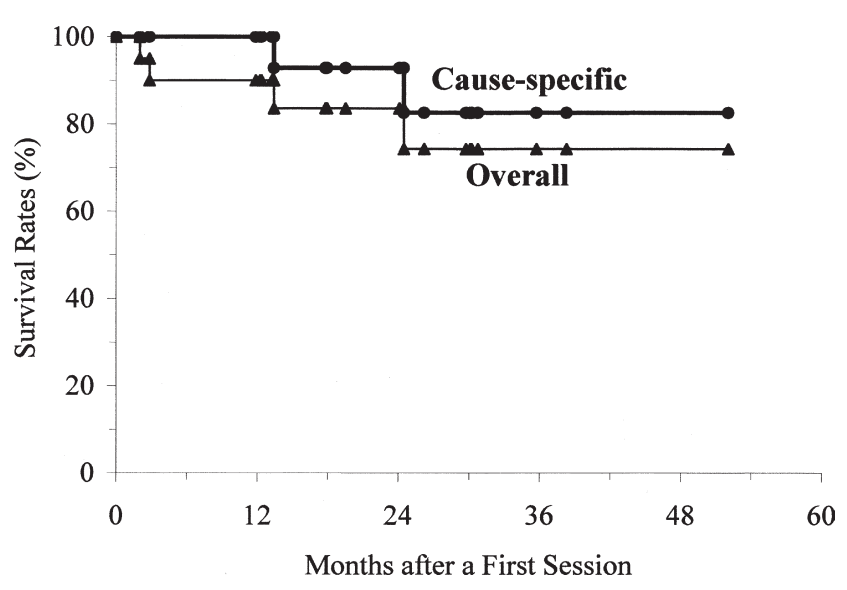

Figure 1. The overall survival and cancer-specific survivals were $90 \%$ and $100 \%$ at 1 year, $84 \%$ and $93 \%$ at 2 years, and $74 \%$ and $83 \%$ at 3 years, respectively.

and has not shown local progression. The local control rates were $72 \%$ at 1 year, $63 \%$ at 2 years, and $63 \%$ at 3 years. Of the 7 patients with local progression, 2 were treated with a total of 3 repeat sessions. Thereafter, local progression was not observed in 1 of the 2 patients. The other 5 patients with local progression were treated with XRT $(\mathrm{n}=1)$, systemic chemotherapy $(\mathrm{n}=3)$ because of coexistent lymphatic and/or hematogenous metastasis, or are awaiting repeat RFA $(\mathrm{n}=1)$.

Of the 20 patients, 4 died at the time of study. Of these, 2 died of cancer progression and the remaining 2 died of other causes; 16 patients survived. Of the 16 patients, 12 were free of recurrence and 4 showed recurrences somewhere. The mean survival time was 42 months; the median survival was not reached. The overall survival and cancerspecific survivals (95\% confidence intervals) were $90 \%$ $(77 \%-100 \%)$ and $100 \%(100 \%-100 \%)$ at 1 year, $84 \%$ $(66 \%-100 \%)$ and $93 \%(79 \%-100 \%)$ at 2 years, and $74 \%$ $(51 \%-97 \%)$ and $83 \%(60 \%-100 \%)$ at 3 years, respectively (Figure 1). The survivals (95\% confidence intervals) with regard to stages IA and IB cancer were 93\% (79\%-100\%) and $83 \%(54 \%-100 \%)$ at 1 year, 93\% (79\%-100\%) and $67 \%(29 \%-100 \%)$ at 2 years, and $80 \%(53 \%-100 \%)$ and unavailable at 3 years, respectively (Figure 2).

\section{Discussion}

RFA appears to be a promising treatment modality for hepatocellular and renal cell carcinoma in terms of local control and patient survival. The local recurrence rate was $14.9 \%(352 / 2369)$ for hepatocellular carcinoma and $0 \%$ to $19 \%$ for renal cell carcinoma. ${ }^{5-7}$ However, it should be noted that NSCLC exhibits a different biologic behavior from those cancers. NSCLC frequently shows microscopic invasion of the lymphatic vessels and finally metastasizes to

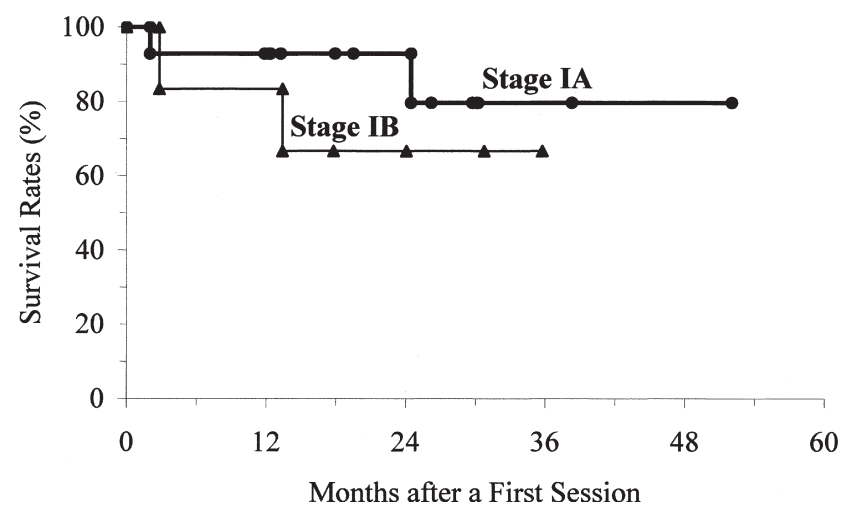

Figure 2. The survivals with regard to stages IA and IB cancer were $93 \%$ and $83 \%$ at 1 year, $93 \%$ and $67 \%$ at 2 years, and $80 \%$ and unavailable at 3 years, respectively.

the regional lymph nodes. In fact, pathologic examinations demonstrated that approximately $20 \%$ of even small $(\leq 2$ $\mathrm{cm}$ in diameter) NSCLCs showed lymph node metastasis. ${ }^{8}$ Because RFA represents only a local therapy, a higher risk of locoregional recurrence and thereby poorer survival is expected as compared with the use of lobectomy accompanied by systematic lymph node sampling or dissection. In the present study, therefore, application of this therapy was limited to the patients who had inoperable disease or who refused surgery.

An international survey reported that RFA of lung tumors is a safe procedure with an extremely low mortality rate $(0.4 \%){ }^{9}$ The most common complication is pneumothorax, but the majority of patients are treated conservatively. ${ }^{9,10}$ Although mid- to long-term local control remains poorly understood, most of the previous studies with a mean follow-up of 1 year or more have shown a primary local control rate of approximately $60 \%$ to $70 \%,{ }^{11-14}$ which is applicable in our study. The local control outcomes of lung cancers seem inferior to those of hepatocellular and renal cell carcinoma, which may be explained by the following reasons: microscopic tumor extension and the difficulty in achieving an adequate treatment margin owing to the severely limited electrical and thermal conductivity of the air-containing lung tissues. Compared with the outcomes after surgical resection of stage I NSCLC, the local progression rate of $35 \%$ in our study is much higher. One of the important limitations of RFA is the difficulty of realizing the precise extent of treatment zone during the procedure; this likely contributes to the relatively high local progression rate. Appearance of ground-glass opacity surrounding treated tumors might aid in recognizing the extent of ablation zone, but it does not indicate the exact extent of coagulation necrosis. $^{15}$

Although the literature contains only limited survival data after RFA of NSCLC, Ambrogi and colleagues ${ }^{13}$ 
reported the outcomes in 40 patients with NSCLC (unknown staging): a mean survival time of 19 months and survivals of approximately $70 \%$ at 1 year, approximately $45 \%$ at 2 years, and $30 \%$ at 3 years. Thanos and associates ${ }^{16}$ reported survivals of approximately $80 \%$ at 1 year, approximately $65 \%$ at 2 years, and approximately $10 \%$ at 3 years in 14 patients with stage II-IV cancer. Fernando and colleagues ${ }^{12}$ reported a survival of approximately $80 \%$ at 2 years in 18 patients including 9 patients with stage I cancer. Simon and colleagues ${ }^{17}$ reported survivals of $78 \%, 57 \%, 36 \%$, and $27 \%$ at $1,2,3$, and 5 years, respectively, in 75 patients with stage I NSCLC (stage IA, $\mathrm{n}=56$; stage IB, $\mathrm{n}=19$ ). Although the survival data in our study appear to be better than the aforementioned data, a relatively high rate of including the patients who were operable but refused surgery or those who had lesions showing pure ground-glass opacity might have contributed to this better survival. As expected, the survival in patients with stage IA cancer seemed better than that of patients with stage IB cancer in our study, but a meaningful comparison analysis was, regrettably, not allowed because of the small study population.

Nonsurgical candidates with NSCLC are generally treated with conventional XRT. In a study on 71 patients with node-negative NSCLC who underwent XRT, overall and disease-specific survivals were $19 \%$ and $44 \%$ at 3 years, respectively. ${ }^{18}$ Another study reported a local recurrence rate of 53\% and the median survival of 20 months for 60 patients with stage I or II cancer who underwent XRT. ${ }^{19}$ Meta-analysis of stage I NSCLC treated by conventional XRT revealed mean overall and cause-specific survivals at 3 years of $34 \%$ and $39 \%$, respectively. ${ }^{20}$ Stereotactic radiotherapy, a novel radiation therapy, shows favorable local control and survivals and may rival RFA. For stage I cancer, the local recurrence rate ranged from $2 \%$ to $20 \%$ at a median follow-up period of longer than 1 year ${ }^{21-24}$; survivals were $80 \%$ or more at 1 year, ${ }^{22-24} 58 \%$ to $75 \%$ at 2 years, ${ }^{22-24}$ and $55 \%$ at 3 years. ${ }^{22}$ In future, it would be interesting to compare RFA with XRT including stereotactic radiotherapy in a randomized controlled study.

The challenging issue after RFA may be the radiologic evaluation of local efficacy. Unlike surgical resection, the ablated tumor remains in situ and thereby assessment of local efficacy is more difficult. The tissue surrounding a tumor that undergoes coagulation necrosis may increase the size of the imaging abnormality immediately after RFA. This phenomenon may cause a tumor that has been completely ablated to appear to have grown. On subsequent follow-up, lesions visualized on CT imaging frequently do not undergo significant shrinkage, even if no viable tissue is present. Therefore, tumor size after RFA does not exactly correlate with tumor viability, and thus we evaluated local efficacy with the aid of contrast enhancement. However, it is true that contrast enhancement of local progression was not always sufficiently clear to make judgments because NSCLC is originally not so hypervascular. PET might be more useful for evaluation; however, its role has not yet been determined. As well as the potential, PET might have a limitation in that inflammation induced by RFA may result in false positive results, especially in the early period after RFA. Regarding biopsy, we suggest that it is not a suitable routine follow-up modality because it is invasive and involves risks such as pneumothorax, hemothorax, and systemic air embolism. Further, considering that the treated tumor contains necrotic tissue, even if it is not completely treated, a small specimen obtained by biopsy may result in a high false negative rate. Thus, we performed it only in selected cases.

The restricted ablation volume is clearly an important limitation of RFA. Lung tumors larger than $3 \mathrm{~cm}$ are at a higher risk of local recurrence than smaller tumors. ${ }^{6,9}$ Dupuy and coworkers ${ }^{25}$ performed RFA followed by conventional XRT in 24 patients with stage I cancer (mean size, $3.4 \mathrm{~cm})$. The local progression rate of $8.3 \%(2 / 24)$ at the mean follow-up of 26.7 months appeared quite promising given the large tumor sizes and longer follow-up in their study. Such favorable outcomes encourage combining XRT and RFA, especially for treating larger tumors. Combination of RFA with chemotherapy may be another option to improve the outcomes. In an animal experiment, RFA followed by intravenous chemotherapy provided larger coagulation than RFA alone. ${ }^{26}$ Systemic chemotherapy may also facilitate treatment of microscopic metastasis that possibly occurs in clinical stage I cancer.

Rapid development of technology will improve the current RFA system to provide more predictable and consistent coagulation and thereby minimize local progression. In animal studies, successful enlarged coagulation necrosis was obtained by various attempts, including modulation of conductivity, ${ }^{27}$ blood perfusion, ${ }^{28}$ and ventilation. ${ }^{29}$ Thus, application of these techniques to the clinical setting could possibly improve local control. Our outcomes might be impaired owing to the use of internally cooled electrodes in the early part of our study because internally cooled electrodes exhibited inferior local control as compared with multitined expandable electrodes. ${ }^{14}$ Anyhow, RFA has just emerged in the treatment of lung cancer, and much work will be done to enhance its efficacy. Notable advantages of RFA include the low invasiveness, preservation of pulmonary function, ${ }^{13}$ the freedom to perform the procedure regardless of any previous therapy, and the availability to repeat procedures whenever required. Therefore, despite the inherent limitation of local therapy in the treatment of NSCLC, RFA may have the potential to play an important role in the treatment of localized cancer.

Our study had several limitations. This is a retrospective review, possibly with selection biases. The biases may make 
it difficult to compare our data with the data obtained in other studies and to draw a conclusion regarding the role of RFA in the treatment of NSCLC. Treatment parameters such as ablation device and algorithm were heterogeneous. PET was not used for radiologic assessment of the disease in most patients. Over the past years, the use of PET has significantly improved nonsurgical staging. ${ }^{30}$ The limited use of PET and the unavailability of mediastinoscopy for preprocedural cancer evaluation might affect the reliability of cancer staging in our study. Inasmuch as the rationale for application of local therapy is based on localization of the disease, candidates for RFA should be appropriately selected by use of the most reliable modality. The survivals of patients in the present study were promising, but the study was carried out on a small study population. Currently, a prospective study on RFA of stage I NSCLC with the use of uniform ablation parameters, a combination of XRT and chemotherapy for selected cancers, and PET scan for radiologic assessment is in progress at our institution.

In conclusion, treating clinical stage I NSCLC with one or more RFA sessions provided promising outcomes in terms of survival. However, considering the relatively high local progression rate, RFA should be considered only for nonsurgical candidates.

\section{References}

1. Ginsberg RJ, Rubinstein LV. Randomized trial of lobectomy versus limited resection for T1 N0 non-small cell lung cancer. Ann Thorac Surg. 1995;60:615-23.

2. Yasui K, Kanazawa S, Sano Y, Fujiwara T, Kagawa S, Mimura H, et al. Thoracic tumors treated with CT-guided radiofrequency ablation: initial experience. Radiology. 2004;231:850-7.

3. Lee JM, Jin GY, Goldberg SN, Lee YC, Chung GH, Han YM, et al. Percutaneous radiofrequency ablation for inoperable non-small cell lung cancer and metastases: preliminary report. Radiology. 2004;230: 125-34.

4. Steinke K, King J, Glenn D, Morris DL. Radiologic appearance and complications of percutaneous computed tomography-guided radiofrequency-ablated pulmonary metastases from colorectal carcinoma. J Comput Assist Tomogr. 2003;27:750-7.

5. Mulier S, Ni Y, Jamart J, Ruers T, Marchal G, Michel L. Local recurrence after hepatic radiofrequency coagulation: multivariate meta-analysis and review of contributing factors. Ann Surg. 2005;242: 158-71.

6. Farrell MA, Charboneau WJ, DiMarco DS, Chow GK, Zincke H, Callstrom MR, et al. Imaging-guided radiofrequency ablation of solid renal tumors. AJR Am J Roentgenol. 2003;180:1509-13.

7. Mayo-Smith WW, Dupuy DE, Parikh PM, Pezzullo JA, Cronan JJ. Imaging-guided percutaneous radiofrequency ablation of solid renal masses: techniques and outcomes of 38 treatment sessions in 32 consecutive patients. AJR Am J Roentgenol. 2003;180: 1503-8.

8. Asamura H, Nakayama H, Kondo H, Tsuchiya R, Shimosato Y, Naruke T. Lymph node involvement, recurrence, and prognosis in resected small, peripheral, non-small cell lung carcinomas: are these carcinomas candidates for video-assisted lobectomy? J Thorac Cardiovasc Surg. 1996;111:1125-34.

9. Steinke K, Sewel PE, Dupuy D, Lencioni R, Helmberger T, Kee ST, et al. Pulmonary radiofrequency ablation: an international study survey. Anticancer Res. 2004;24:339-44.
10. Hiraki T, Tajiri N, Mimura H, Yasui K, Gobara H, Mukai T, et al. Pneumothorax, pleural effusion, and chest tube placement after radiofrequency ablation of lung tumors: incidence and risk factors. Radiology. 2006;241:275-83.

11. Steinke K, Glenn D, King J, Clark W, Zhao J, Clingan P, et al. Percutaneous imaging-guided radiofrequency ablation in patients with colorectal pulmonary metastases: 1-year follow-up. Ann Surg Oncol. 2004;11:207-12.

12. Fernando HC, De Hoyos A, Landreneau RJ, Gilbert S, Gooding WE, Buenaventura PO, et al. Radiofrequency ablation for the treatment of non-small cell lung cancer in marginal surgical candidates. $J$ Thorac Cardiovasc Surg. 2005;129:639-44.

13. Ambrogi MC, Lucchi M, Dini P, Melfi F, Fontanini G, Faviana P, et al. Percutaneous radiofrequency ablation of lung tumours: results in the mid-term. Eur J Cardiothorac Surg. 2006;30:177-83.

14. Hiraki T, Sakurai J, Tsuda T, Gobara H, Sano Y, Mukai T, et al. Risk factors for local progression after percutaneous radiofrequency ablation of lung tumors: evaluation based on a preliminary review of 342 tumors. Cancer. 2006;107:2873-80.

15. Yamamoto A, Nakamura K, Matsuoka T, Toyoshima M, Okuma T, Oyama Y, et al. Radiofrequency ablation in a porcine lung model: correlation between CT and histopathologic findings. AJR Am J Roentgenol. 2005; 185:1299-306.

16. Thanos L, Mylona S, Pomoni M, Athanassiadi K, Theakos N, Zoganas L, et al. Percutaneous radiofrequency thermal ablation of primary and metastatic lung tumors. Eur J Cardiothoracic Surg. 2006;30:797-800.

17. Simon CJ, Dupuy DE, DiPetrillo TA, Safran HP, Grieco CA, Ng T, et al. Pulmonary radiofrequency ablation: long-term safety and efficacy in 153 patients. Radiology. 2007;243:268-75.

18. Kupelian PA, Komaki R, Allen P. Prognostic factors in the treatment of node-negative non-small cell lung carcinoma with radiotherapy alone. Int J Radiat Oncol Biol Phys. 1996;36:607-13.

19. Zierhut D, Bettscheider C, Schubert K, van Kampen M, Wannenmacher M. Radiation therapy of stage I and II non-small cell lung cancer (NSCLC). Lung Cancer. 2001;34:39-43.

20. Qiao X, Tullgren O, Lax I, Sirzen F, Lewensohn R. The role of radiotherapy in treatment of stage I non-small cell lung caner. Lung Cancer. 2003;41:1-11.

21. Nagata Y, Takayama K, Matsuo Y, Norihisa Y, Mizowaki T, Sakamoto T, et al. Clinical outcomes of a phase I/II study of $48 \mathrm{~Gy}$ of stereotactic body radiotherapy in 4 fractions for primary lung cancer using a stereotactic body frame. Int J Radiat Oncol Biol Phys. 2005; $63: 1427-31$

22. Nyman J, Johansson KA, Hulten U. Stereotactic hypofractionated radiotherapy for stage I non-small cell lung cancer: mature results for medically inoperable patients. Lung Cancer. 2006;51:97-103.

23. Ohishi H, Kuriyama K, Komiyama T, Tanaka S, Sano N, Marino K, et al. Clinical outcomes of stereotactic radiotherapy for stage I non-small cell lung cancer using a novel irradiation technique: patient self-controlled breath-hold and beam switching using a combination of linear accelerator and CT scanner. Lung Cancer. 2004;45:45-55.

24. Zimmermann FB, Geinitz H, Schill S, Grosu A, Schratzenstaller U, Molls M, et al. Stereotactic hypofractionated radiation therapy for stage I non-small cell lung cancer. Lung Cancer. 2005;48:107-14.

25. Dupuy DE, DiPetrillo T, Gandhi S, Ready N, Ng T, Donat W, et al. Radiofrequency ablation followed by conventional radiotherapy for medically inoperable stage I non-small cell lung cancer. Chest. 2006; 129:738-45.

26. Goldberg SN, Kamel IR, Kruskal JB, Reynolds K, Monsky WL, Stuart $\mathrm{KE}$, et al. Radiofrequency ablation of hepatic tumors: increased tumor destruction with adjuvant liposomal doxorubicin therapy. AJR Am J Roentgenol. 2002;179:93-101.

27. Lee JM, Youk JH, Kim YK, Han YM, Chung GH, Lee SY, et al. Radio-frequency thermal ablation with hypertonic saline solution injection of the lung: ex vivo and in vivo feasibility studies. Eur Radiol. 2003;13:2540-7. 
28. Hiraki T, Gobara H, Sakurai J, Mimura H, Mukai T, Hase S, et al. Radiofrequency ablation of normal lungs after pulmonary artery embolization with use of degradable starch microspheres: results in a porcine model. J Vasc Interv Radiol. 2006;17:1991-8.

29. Oshima F, Yamakado K, Akeboshi M, Takaki H, Nakatsuka A, Makita $\mathrm{M}$, et al. Lung radiofrequency ablation with and without bronchial occlusion: experimental study in porcine lungs. $J$ Vasc Interv Radiol. 2004; $15: 1451-6$.

30. Lardinois D, Weder W, Hany TF, Kamel EM, Korom S, Seifert B, et al. Staging of non-small cell lung cancer with integrated positronemission tomography and computed tomography. $N$ Engl J Med. 2003;348:2500-7. 